\title{
Reliability of External Ear Measurements Obtained by Direct, Photocopier Scanning and Photo Anthropometry
}

\author{
Bor-Shong Liu ${ }^{\dagger}$ \\ Department of Industrial Engineering and Management \\ St. John's University Tamsui Taipei 25135, Taiwan \\ Tel: +886-2-28013131x6560, E-mail: bsliu@mail.sju.edu.tw \\ Hsien-Yu Tseng \\ Department of Industrial Engineering and Management \\ St. John's University Tamsui Taipei 25135, Taiwan \\ E-mail: hytseng@mail.sju.edu.tw \\ Tung-Chung Chia \\ Department of Business Administration \\ Ling Tung University Taichung 40852, Taiwan \\ E-mail: iechia@mail.ltu.edu.tw
}

Received, June 12, 2009; Revised, September 28, 2009 \& January 15, 2010; Accepted, January 18, 2010

\begin{abstract}
The purpose of this study was to compare the consistency of external ear measurements obtained by electronic digital caliper, photocopier scanning and digital photographic methods. Photogrammetric measurements were made after image editing software was used to optimize the brightness, contrast, size and image clarity. The CorelDRAW dimension tool was used to create a dimension line that measured the vertical and horizontal length between any two landmarks. Results of repeated measures analysis of variance revealed no significant differences in length and width of the pinna between the three methods. The reliability of the three measurement techniques showed a high degree of consistency. Further study and efforts could be extended to measurement hands, foots and facial dimensions by present techniques.
\end{abstract}

Keywords: Anthropometry, Ear Dimensions, Reliability, Photogrammetry, Intra-Class Correlation Coefficient

\section{INTRODUCTION}

Anthropometry (measurement of humans), in physical anthropology, refers to the measurement of living human individuals for the purposes of understanding human physical variation. Anthropometry plays an important role in industrial design, clothing design, ergonomics, and architecture, where statistical data about the distribution of body dimensions in the population are used to optimize products. As products are designed for specific types of consumers, an important design requirement is selection and efficient utilization of the most appropriate anthropometric database (Wickens et al., 2004). Anthropometry data provides a valuable source of information to ergonomists and designers who attempt to consider a range of body sizes and abilities in the design of occupa- tional environments and products. An anthropometric measurement is simply a construction of an observation or recording of an attribute, which can be affected by the measurers' characteristics, methods used in measurement and the measurement environment (Feathers et al., 2004).

Several different tools are used for the measurement of anthropometric dimensions. In general, these can be divided into direct and indirect methods (Hsu et al., 2005; Wickens et al., 2004). Direct methods are those that require direct contact of the human body with the measuring tools (e.g., straightedge, measuring tape, Martin-type anthropometer), and direct reading of the indictors. Direct measurements using classical methods of physical anthropology have been used to assess the dimensions, location and level of the external ear in sub-

$\uparrow$ : Corresponding Author 
jects and for patients who have had plastic surgery (Farkas, 1981). A survey of anthropometry aimed to determine the mean values of external ear in Turkish adult population. Seven surface measurements were taken directly from ear of the subjects with electronic digital caliper (Bozkir et al., 2006). Barut and Aktunc (2006) also applied the digital compass to measure the external ear in Turkish primary school students. Direct methods have the advantage that equipment is simple and cheap. Dimensions of survey are usually measured twice by measuring tools and the arithmetical mean is then computed. The adequate use of these tools, however, is often limited by the time-consuming and strenuous physical circumstances of the information gathering process. In addition, direct measurement of a subject's ear can be difficulty, because of distortion of soft tissues of the natural ear during measurement and the problem of the subject in maintaining the orientation of the head for several minutes, which may alter the operator's perspective (Alexander and Laubach, 1968).

Indirect methods can be defined as measurements of a subject obtained without requiring his or her presence. These methods use projection, photography, scanning dimensions of measurements or reference landmarks from images or electronic files, and transform data using scales or mathematical computations of the relevant reference symbols to obtain anthropometric dimensions. Anthropometric systems used in indirect methods typically employ a transparent grid board that has already indicated the size, photogrammetry, and perspective. In addition, Nechala et al. (1999) reported that anthropometric measurements available on the face can be gauged with photogrammetry. The dimensions of face were including upper face height, intercanthal width, nose height etc. In addition, Coward et al. (1997) applied the laser scanner that developed at University College London for capturing an image of the face. These systems have some inherent advantages including (1) no need for direct contact with the human, and ability to obtain dimensions by photogrammetry or optical scanning alone; (2) relatively short exam time for subjects; (3) allows subjects to relax because of easy data collection; (4) the images or electronic files can be reviewed or re-measured as needed. By contrast, disadvantages of indirect methods include: (1) equipment uses more expensive software and hardware; (2) installation, use and maintenance of the equipment requires more greater technological skill; (3) environmental conditions of the equipment require stricter control, and mobility is restricted; (4) while photographing or scanning, some areas of body must be covered, making points in these areas more difficult to measure. Thus, images may require correction or re-processing.

Using two-dimensional imaging techniques could be obtaining distance data. With the advent of digital photography and digital photograph manipulation, lowcost imaging techniques would be developing for obtaining anthropometrical data. For example, images were from photocopier scanning and from digital photographs.
Although a vast number of studies have reported anthropometric data, few have investigated the reliability of results from different obtaining methods. Studies are needed to develop error checking mechanisms for different conditions, postural considerations, and measurement approaches. The purpose of this study was to compare the reliability of measurements of external ear dimensions obtained by direct measurement, by scanning from a photocopier and from digital photographs. Possible error sources were also investigated.

\section{METHODS}

\subsection{Participants}

Thirty healthy subjects with normal ears (18 men and 12 women) were included in this study. Their age ranged from 20 to 47 years old. The mean height was $169.6 \mathrm{~cm}(\mathrm{SD}=8.15)$ and mean weight was $64.7 \mathrm{~kg}(\mathrm{SD}$ $=13.23$ ). Only the left ear of participants was studied.

\subsection{External Ear Landmarks and Their Measurement}

Ear-structure and the related knowledge about the ears were studied by the investigators before any subject was surveyed. Investigators reviewed the position, shape and measurement methods and the relation between the landmarks. The length and width of pinna were defined as follows:

(1) Pinna length: the distance between the top of the helix and the bottom lobule of the pinna.

(2) Pinna width: the largest distance between the left and right edge of the pinna on the mid-sagittal plane.

\subsection{Direct Anthropometry}

Subjects were asked to pose in the sitting position in front of a white sheet or plain wall with the head held so that they looked straight forward with the lower borders of the eye sockets in the same horizontal plane as the external auditory meati (i.e. Frankfurt plane). The chin position and head tilt were then adjusted until the head was correctly aligned. This procedure was checked several times as necessary before the final measurement was taken. After confirming the posture of subject, two dimensions of the left external ear were measured with an electronic digital caliper Figure 1. Only one trained measurer performed the all measurements by three times and time spaced between any two measurements was one week.

\subsection{Photocopier Scanning}

The subject adopted a standing position with the left ear on the scanning faceplate. Subject's left ear slightly contact with scanning faceplate and gauge block of 10 $\mathrm{mm}$ placed on head near upper pinna to avoid deforming 


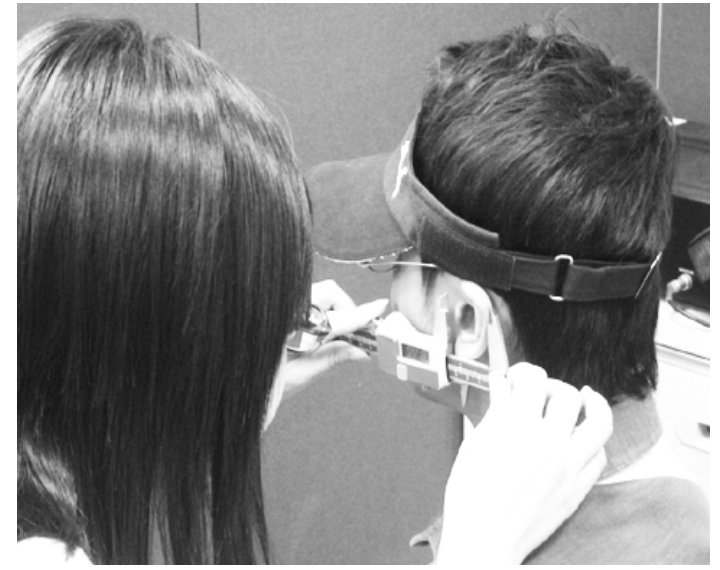

Figure 1. Measurement of what anatomical feature or landmark by direct anthropometry.

overly of the ear. Naturally subjects closed their eyes. The photocopier (Gestetner MP 2550, Ricoh) had been set full-size and high photo quality. After printing the hardcopy, two dimensions of the external ear were also measured with a digital caliper on hardcopy Figure 2 and Figure 3. In addition, total measures of three times were performed between a week.

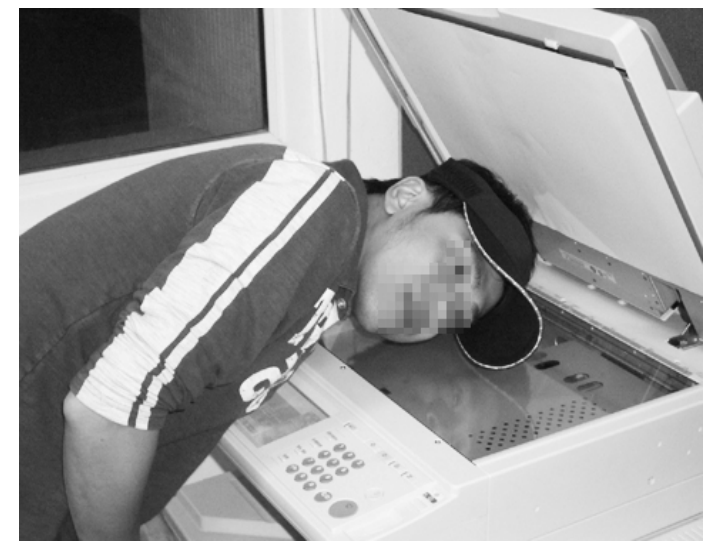

Figure 2. Scanning of the left ear of a subject with a photocopier.

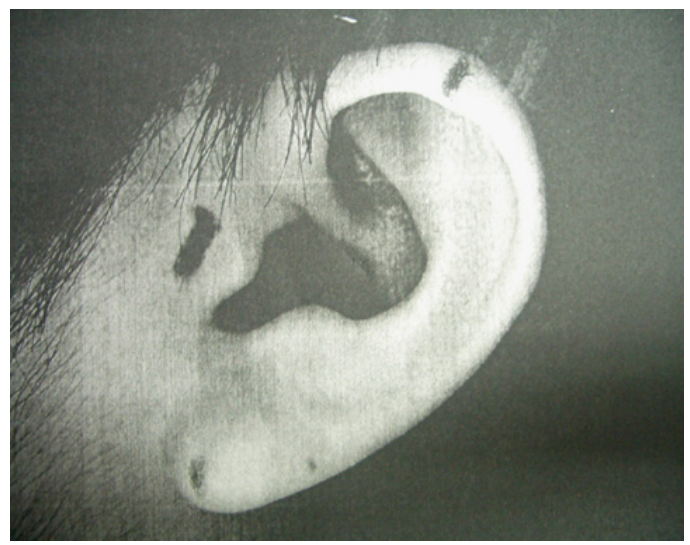

Figure 3. Image of left ear scanned from a photocopier.

\subsection{Photogrammetric Measurements}

Both the camera lens and the photographer were aligned and parallel to the subject to reduce the possibility of image perspective distortion due to poor positioning Figure 4. The mid-vertical grid line of the camera viewfinder was aligned to pass through the mid-sagittal plane of the face. The mid-horizontal passed through the Frankfurt horizontal plane. Ear features were then captured using a digital camera (Panasonic DMC-LX2GT, $3168 \times 2376$ pixels) mounted on a tripod and profile views were taken at a distance of $90 \mathrm{~cm}$ between the subject and lens. A gauge block (10 mm, Mitutoyo, Japan) was used for calibration. In addition, total measures of three times were performed between a week.

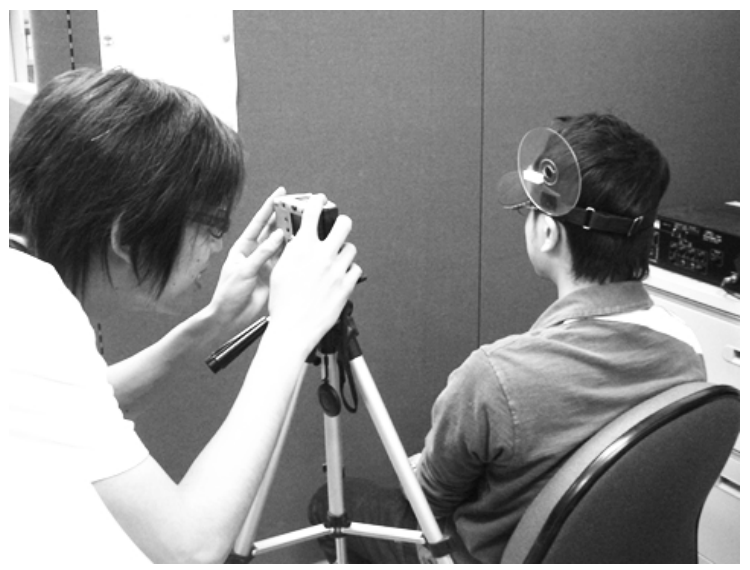

Figure 4. Set up for photogrammetric technique.

\subsection{Image Editing of Photogrammetric Measurements}

Accurate identification of landmarks on images of the ear is difficult. Therefore, photo images were edited to provide clear landmarks to improve measurement accuracy. Images were modified using the editing tools and techniques in Adobe Photoshop (Adobe Systems Inc.) before measuring the dimensions with CorelDraw. First, the images were converted to grayscale. Second, images were modified by using the uniform distribution command. Finally, the curves command was used to adjust the tonal range of an image. These details of these techniques were as follows:

(1) De-saturation: the image was converted to grayscale using the saturation command, but each pixel of image was kept at the same level Figure 5a.

(2) Uniform distribution: this command redistributes the brightness level of image pixels (ranged from 0 to 255), and enables presentation of an entirely equal brightness level. On executing this command, Photoshop detects the brightest and the darkest values in the image, and then assigns the brightest and the darkest value to be 255 and 0 , respectively. After applying the uniform distribution, the image has a 
shaper and clearer appearance Figure 5b.

(3) Curves: Instead of making adjustments using only three variables (highlights, shadows, and midtones), the curves command adjusts the highlights and shadows in opposite directions to boost contrast. Using this command the curves of the images were manipulated until the highest contrast was obtained.

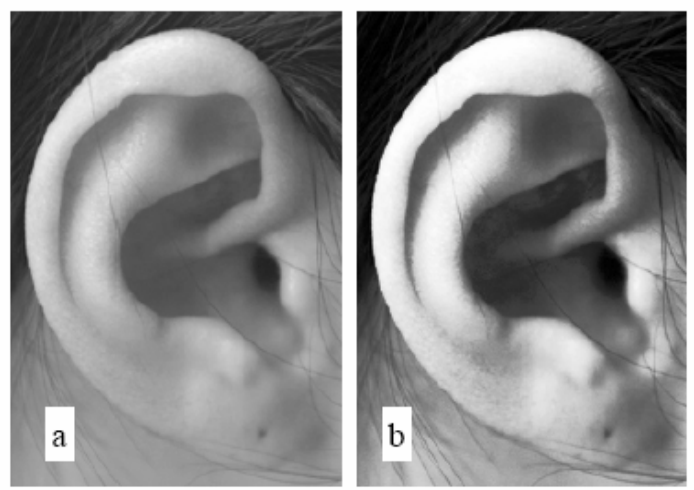

Figure 5. (a) Color de-saturated image; (b) Image after uniform distribution.

\subsection{Photogrammetric Measurement of Dimensions}

After the photograph was taken, a suitable size and editing of the image was performed with the Photoshop 7.0. This software was used to optimize the brightness, contrast, size and produce a clear image. The CorelDRAW dimension tool was then used to create a vertical dimension line that measured the vertical distance between any two landmarks along the $y$-axis. (CorelDRAW X3, Corel Corporation). This method allowed ear dimensions to be easily and accurately calculated by comparing the two landmarks Figure 6. Finally, the two dimensions of ear could be calibration by gauge block (10 $\mathrm{mm})$.

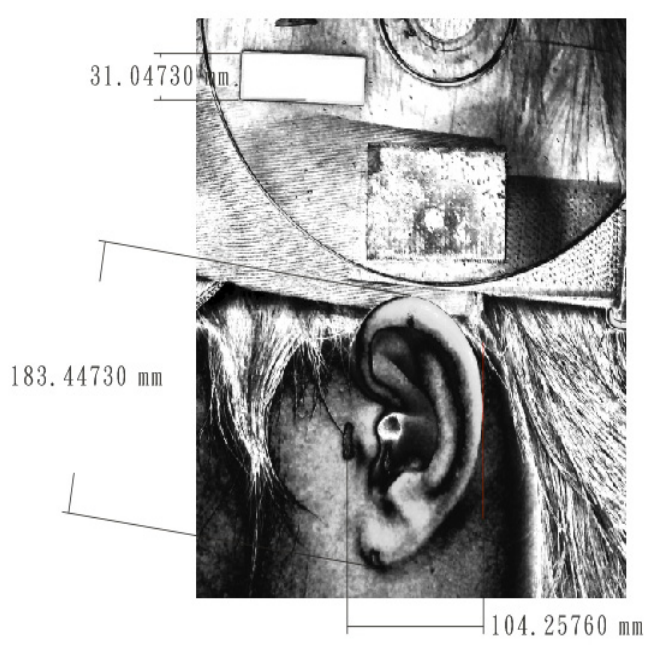

Figure 6. Measuring dimensions with CorelDraw.

\subsection{Data Analysis}

After data coding, repeated measures analysis of variance (ANOVA) was performed with statistical analysis software (Norusis, 2004). Repeated measures analysis deals with response outcomes measured on the same experimental unit at different times or under different conditions, and the total variation is partitioned into the following three components: (1) variation among individuals; (2) variation among test occasions; (3) residual variation (Hinkle et al., 2003; Lindsey, 1993). In addition, the intra-class correlation coefficient (ICC) has been used as a measure of internal consistency, representing the percentage of variance explained by individuals (McGraw and Wong, 1996). Further, the precision of the interrater measurements of pinna dimensions was assessed by calculating the standard error of measurements (SME). The SEM was calculated as:

$$
S E M=s d \times \sqrt{(1-I C C)},
$$

where ICC is the reliability coefficient and sd is the square root of the total variance. The SEM can be used to establish statistically meaningful levels of change for a measurement (Portney and Watkins, 1993).

\section{RESULTS}

\subsection{Repeated Measures Analysis}

Results of repeated ANOVA on pinna length revealed no significant differences between the three methods $(F(2,87)=0.225, P=0.799)$. In addition, these were also no significant differences between the three measurements $(\mathrm{F}(2,174)=0.480, \mathrm{P}=0.620)$. The results of analysis of pinna width showed remarkable consistency with those of pinna length. There were not significant differences between results obtained by the three methods $(\mathrm{F}(2,87)=0.140, \mathrm{P}=0.870)$ or for the three measurements $(\mathrm{F}(2,174)=2.857, \mathrm{P}=0.060)$. However, an interaction between methods and time was found for the measurement of pinna width $(\mathrm{F}(4,174)=$ $2.518, \mathrm{P}=0.043)$. Figure 7 shows the interaction between method and time in the measurement of pinna width. The symbols $\mathrm{W} 1, \mathrm{~W} 2$, and $\mathrm{W} 3$ on the horizontal axis denote the first, second and third measurement. Further, measurements obtained from photocopier scans and photogrammetric study did not significantly vary with time. By contrast, direct anthropometry results showed a slight decline on W2 and W3. Because pinna is the plastic tissue, deforming of the ear might be difference in each direct measurement by electronic digital caliper. However, images scanning from photocopier, subject's left ear slightly contact with scanning faceplate and gauge block of $10 \mathrm{~mm}$ placed on head near upper pinna to avoid deforming overly of the ear. In addition, the 
deforming of the ear did not be occurred in photogrammetric measurements.

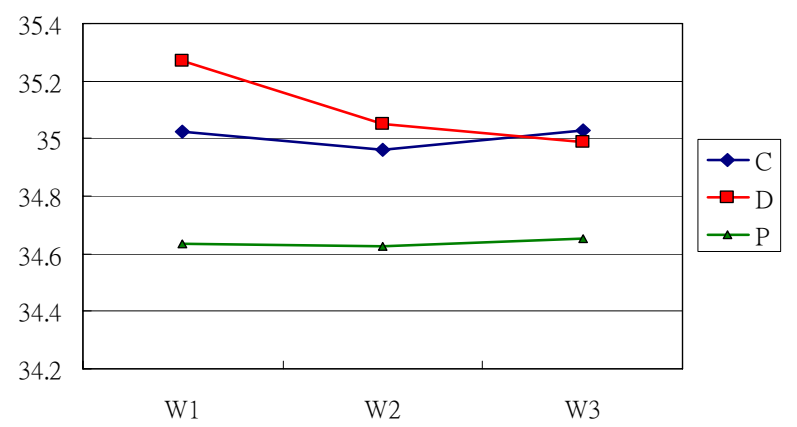

Figure 7. Interaction effect between method and time in pinna width measurement. C: Scanning From Photocopier; D: Direct Anthropometry; P: Photogrammetric.

\subsection{Reliability Analysis}

It is current practice to accept ICC values in the range of $0.8-1.0$ as excellent repeatability, 0.6-0.8 as good repeatability, and values below 0.6 as poor repeatability (McGraw and Wong 1996).

Analysis of our recorded pinna length measurements showed the three methods all had excellent reliability (Table 1). In addition, summary statistics for the three methods revealed that the range of measurement results was higher for direct measurement (0.19) than for scanning (0.01) and photogrammetric measurement (0.09). Further, the variances were the lowest for scan- ning measurement methods indicating that this was a more consistent and reliable method. The SEM values for pinna length measurement by the direct, scanning and photogrammetric methods were $0.6,0.15$ and 0.37 $\mathrm{mm}$, respectively. These findings suggest that when researchers repeat the measure of pinna length by direct methods in the same subject on different occasions, such as pre and post manipulative intervention, and the measurement changes less than $0.6 \mathrm{~mm}$, the difference may be attributable to measurement error.

For pinna width measurement, all three methods also had excellent reliability (Table 2). The range of measurement results was greater in direct measurement (0.28) than for scanning (0.07) and photogrammetric measurements $(0.03)$. In addition, comparison of measurements between pinna length and width revealed dramatically lower variances for pinna width measure-ment by direct anthropometry compared to the other methods. Further, the SEM values for pinna length measurement by direct, scanning and photogrammetric method were $0.34,0.24$ and $0.29 \mathrm{~mm}$, respectively.

\section{DISCUSSION}

Applied anthropometric or morphometry of external ear were most conducted in aesthetic plastic surgery. Farkas et al. (1992) analyzed by measuring the width and length of ears in 1,590 North American Caucasians between 1 year and 18 years of age. Results also reported that mature width of the ear is achieved in males at 7 years and in females at 6 years and ear length matured in males at 13 years and in females at 12 years. In addition, Bozkir et al. (2006) were taken the dimensions of external ear from 341 healthy Turkish young adults

Table 1. Reliability analysis of pinna length measurements obtained by different methods.

\begin{tabular}{|c|c|c|c|c|c|c|c|c|c|}
\hline Methods & & Mean & Min & Max & Range & $\mathrm{N}$ & $\mathrm{ICC} \%$ & $\begin{array}{c}\text { 95\% Conference } \\
\text { Interval }\end{array}$ & $\begin{array}{l}\text { SEM } \\
(\mathrm{mm})\end{array}$ \\
\hline \multirow{2}{*}{ Direct } & Mean & 61.43 & 61.32 & 61.51 & 0.19 & \multirow{2}{*}{30} & \multirow{2}{*}{98.8} & \multirow{2}{*}{ 97.8-99.4 } & \multirow{2}{*}{0.6} \\
\hline & Variance & 30.97 & 29.87 & 33.02 & 3.15 & & & & \\
\hline \multirow{2}{*}{ Scanning } & Mean & 60.67 & 60.66 & 60.67 & 0.01 & \multirow{2}{*}{30} & \multirow{2}{*}{99.9} & \multirow{2}{*}{$99.9-100$} & \multirow{2}{*}{0.15} \\
\hline & Variance & 24.43 & 24.25 & 24.57 & 0.32 & & & & \\
\hline \multirow{2}{*}{ Photo } & Mean & 60.57 & 60.51 & 60.60 & 0.09 & \multirow{2}{*}{30} & \multirow{2}{*}{99.6} & \multirow{2}{*}{$99.2-99.8$} & \multirow{2}{*}{0.37} \\
\hline & Variance & 34.95 & 32.82 & 36.51 & 3.69 & & & & \\
\hline
\end{tabular}

Table 2. Reliability analysis on pinna width measurements obtained by different methods.

\begin{tabular}{|c|c|c|c|c|c|c|c|c|c|}
\hline Methods & & Mean & Min & Max & Range & $\mathrm{N}$ & $\mathrm{ICC} \%$ & $\begin{array}{l}\text { 95\% Conference } \\
\text { Interval }\end{array}$ & $\begin{array}{l}\text { SEM } \\
(\mathrm{mm})\end{array}$ \\
\hline \multirow{2}{*}{ Direct } & Mean & 35.10 & 34.99 & 35.27 & 0.28 & \multirow{2}{*}{30} & \multirow{2}{*}{99.2} & \multirow{2}{*}{$98.5-99.6$} & \multirow{2}{*}{0.34} \\
\hline & Variance & 15.17 & 14.98 & 15.53 & 0.55 & & & & \\
\hline \multirow{2}{*}{ Scanning } & Mean & 35.00 & 34.96 & 35.03 & 0.07 & \multirow{2}{*}{30} & \multirow{2}{*}{99.4} & \multirow{2}{*}{$98.9-99.7$} & \multirow{2}{*}{0.24} \\
\hline & Variance & 9.88 & 9.75 & 10.02 & 0.27 & & & & \\
\hline \multirow{2}{*}{ Photo } & Mean & 34.64 & 34.62 & 34.65 & 0.03 & \multirow{2}{*}{30} & \multirow{2}{*}{99.4} & \multirow{2}{*}{ 98.9-99.7 } & \multirow{2}{*}{0.29} \\
\hline & Variance & 14.14 & 13.43 & 14.76 & 1.33 & & & & \\
\hline
\end{tabular}


(150 female and 191 male) ages 18 to 25 years using an electronic digital caliper. Previous study recorded the three different dimensions of the outer ear from two hundred Taiwanese subjects aged 20 59 and divided into four age stratifications. Results of analysis showed that there were not significant differences in all ear dimensions across age groups, because participant population of study is all adult people (Liu, 2008).

For comparison of external ear dimensions, it was observed that the mean ear length was $62.4 \mathrm{~mm}$ in males and $58.5 \mathrm{~mm}$ in female in North American Caucasians (ages from 1 to 18 years old), and that the same measurements were $63.1 \mathrm{~mm}$ and $59.7 \mathrm{~mm}$ in Turkish (ages from 18 to 25 years old), $71.2 \mathrm{~mm}$ and $65.4 \mathrm{~mm}$ in Korean (mean age 30 years old), respectively (Bozkir et al., 2006; Farkas. 1992; Jung and Jung. 2003). In the current study, the mean pinna length was found to be $64.3 \mathrm{~mm}$ in males and $57.1 \mathrm{~mm}$ in females (ages from 20 to 47 years old). Thus, comparing the ethnic difference in pinna length showed that Korean have larger ears. For pinna width, the mean width was $33.1 \mathrm{~mm}$ in males and $31.2 \mathrm{~mm}$ in females from adult Turkish people. The mean pinna width was $37.1 \mathrm{~mm}$ in males and $32.1 \mathrm{~mm}$ in females. Taiwanese have wider than Turkish.

Measurement consistency can be ascertained by analysis of repeated measurements across time. With continued repeated measurements, the concordant measurement results establish a measure of variability with measurement, measurer and methodology applied across time. Each dimension is obtained from the subject according to a regular measurement procedure such as identification of landmarks, measuring, confirming and recording. But each of these processes is a potential source of error and variation can easily occur among measurers. Gavan (1950) investigated the potential sources of variance within a given measurement, and concluded that consistency increased as the number of measurers decreased, the amount of subcutaneous tissue decreased, the experience of the measurer increased, and as the landmarks were more clearly defined (Gavan, 1950). Klipstein-grobusch, Georg and Boeing (1997) assessed the intra-and inter-interviewer variability both in anthropometric measurements and in estimates of body composition, and the possibility of systematic variation in these measurements due to interviewer differences. This may provide insight into the type and extent of possible measurement error.

Reliability and precision are essential properties of any clinically meaningful measurement or tool. The reliability of a measurement attests to its ability to be recorded consistently within and between different tools. All measurements are prone to systematic error. Systematic errors are biases in measurement which lead to measured values being systematically too high or too low. A systematic error is any biasing effect, in the environment, methods of observation or instruments used, which introduces error into an experiment and is such that it always affects the results of an experiment in the same direction
(Lexwell and Downham, 2005). In the present study, although the reliability of the three measurement techniques showed a high degree of consistency, the direct measurement technique was prone to higher values as compared to the scanning and photogrammetric techniques. A potential error source might be diminished image quality from photo capture. Bozir et al. (2006) showed that the normal ear has an inclination on the face and ear projection (distance from the helix to the processus mastoideus at the tragal level). Thus, distortion may occur from deviations of the orthogonal projection on the lateral view of the captured image.

Random errors are statistical fluctuations (in either direction) in the measured data due to the precision limitations of the measurement device. Random errors usually result from the experimenter's inability to take the same measurement in exactly the same way and obtain exact the same number. Deviations in the selection of landmarks were the greatest contributors to measurement errors. The accurate siting of landmarks is critical to any technique which assesses dimensional measurements. Anthropometry of a subject's ear can be prone to inaccuracy because of distortion of soft tissues of the natural ear during measurement. The landmarks sited on the face and ear could be reliably located and thus be used in further studies to determine dimensional measurements of the ear and its position on the face. Changes in posture between measurements are another source of inconsistent results (Feathers et al., 2004). These errors arise from the difficulty in maintaining the orientation of the head for the several minutes needed to perform assessments, which may alter the operator's perspective. Thus, providing chin support on the table should be aided to maintain the fixed head posture and reduce deviation.

Methods of mapping the face and quantifying facial dimensions have been developed using white light (Bhatia et al., 1994) and laser scanning (Moss et al., 1989). Despite advances in three-dimensional imaging techniques and its many advantages over two-dimensional techniques, the majority of clinicians still rely on two-dimensional techniques. Two-dimensional photography has been the clinical mainstay for several reasons including cost, accessibility, portability, and ease of use (Inoue et al., 1995; Nechala et al., 1999). In this study, we found no significant differences in measurements of the length and width of the pinna between the three methods. In addition, although reproduction accuracy was not significantly different between each method, the standard deviations among the three methods was significantly larger in direct anthropometry than scanning from photocopier and photogrammetric measurements.

For the purpose of generating digital photographs, it is no surprise that the digital camera is best (Nechala et al., 1999). It should be noted that the field of digital photography is changing very rapidly, with technologic advancements resulting in higher quality digital photographs. At the same time, prices are decreasing, thus bringing this technology within reach of more and more 
people. After a photograph is taken, it can be viewed immediately and discarded right away. The photographs are easily downloaded to a computer where they can be viewed, manipulated, edited, and stored. With recent advances in printer technology, digital pictures can be turned into hard copies of very high quality. From the viewpoint of practical implications, digital camera is thus very feasible for use in anthropometry. Siting of landmarks on an image captured by a digital photograph technique has been shown to be accurate in identifying landmarks of the ear.

\section{CONCLUSIONS}

The availability of an anthropometric database is a prerequisite for designers and engineers of products and facilities. It is know that the shape of the ear and the structure of the cartilegenous tissue of the pinna are distinctive. The features of an ear are not expected to be unique to each individual. No commercial systems are available yet and authentication of individual identity based on ear recognition is still a research topic. For design of the ear-related product was necessarily considering the outer-ear dimensions. Thus, provided the anthropometric dimensions of external ears should be conducted continuously and accuracy of data should be examined. The purpose of this study was to compare the reliability of measurements of external ear dimensions obtained by three techniques. The reliability of measurements in photogrammetry could be of the pinna are distinctive. In addition, the results of present study suggest that landmarks can be reliably sited on an image obtained by a digital camera and used to calculate dimensions of the external ear. Deviations in the selection of landmarks were most common contributors to measurement errors.

Further study and efforts are needed: (1) to collect anthropometric data for more populations, (2) to study the reliability of such measurements would be a more specific purpose, for example, to measure the lobule thickness for earrings or ear studs, (3) to analyze the shape of ears to assist in the development of industrial noise protective equipment (earmuffs, earplugs), (4) to establish a database maintenance system for update data. In addition, further study and efforts could be extended to measurement hands, foots and facial dimensions by present techniques.

\section{ACKNOWLEDGMENT}

Authors wish to thank Ms. Rou-Wen Wang for conducting the experiment and data collection.

\section{REFERENCES}

Alexander, M. and Laubach, L. (1968), Anthropometry of the human ear, Aerospace Medical Research Laboratories, Wright Patterson, Ohio.

Barut, C. and Aktunc, E. (2006), Anthropometric measurements of the external ear in a group of Turkish primary school students, Aesthetic Plastic Surgery, 30, 255-259.

Bhatia, G., Vannier, M. W., Smith, K. E., Commean, P. K., Riolo, J., and Young, V. L. (1994), Quantification of facial surface change using a structured light scanner. Plastic and Reconstructive Surgery, 94, 768-774.

Bozkir, M. G., Karakas, P., Yavuz, M., and Dere, F. (2006), Morphometry of the external ear in our adult population, Aesthetic Plastic Surgery, 30, 8185.

Coward, T. J., Watson, R. M., and Scott, B. J. J. (1997), Laser scanning for the identification of repeatable landmarks of the ears and face, British Journal of Plastic Surgery, 50, 308-314.

Farkas, L. G. (1981), Anthropometry of the head and face in medicine, New York: Elservier.

Farkas, L. G., Posnick, J. C., and Hreczko, T. M. (1992), Anthropometric growth study of the ear, Cleft Palate-craiofacial Journal, 29, 324-329.

Feathers, D. J., Paquet, V. L., and Drury, C. G. (2004), Measurement consistency and three-dimensional electromechanical anthropometry, International Journal of Industrial Ergonomics, 33, 181-190.

Gavan, J. A. (1950), The consistency of anthropometric measurements, American Journal of Physical Anthropometry, 8, 417-426.

Hinkle, D. E., Wiersma, W., and Jurs, S. G. (2003), Applied statistics for the behavioral sciences, 5th Edition, Boston: Houghton Mifflin Company.

Hsu, S. H., Peng Y., and Wu, S. P. (2005), Human Factors Engineering, 3rd Edition, Taichung: Tsang Hai Book Publishing.

Inoue, K., Ichikawa, R., Nagashima, M., and Kodama, G. (1995), Sex differences in the shapes of several parts of the young Japanese face, Applied Human Science, 14, 191.

Jung, H. S. and Jung, H. S. (2003), Surveying the dimensions and characteristics of Korean ears for the ergonomic design of ear-related products, International Journal of Industrial Ergonomics, 31, 361-373.

Klipstein-grobusch, K., Georg, T., and Boeing, H. (1997), Interviewer variability in anthropometric measurements and estimates of body composition, International Journal of Epidemiology, 26, S174-S180.

Lexwell, J. E. and Downham, D. Y. (2005), How to assess the reliability of measurements in rehabilitation, American of Physical Medicine and Rehabilitation, 84, 719-723.

Lindsey, J. K. (1993), Models for Repeated Measurements, Oxford: Oxford University Press.

Liu, B. S. (2008), Incorporating anthropometry into design of ear-related products, Applied Ergonomics, 39, $115-121$.

McGraw, K. O. and Wong, S. P. (1996), Forming infer- 
ences about some intraclass correlation coefficients, Psychological Methods, 1, 30-46.

Moss, J. P., Linney, A. D., Grindrod, S. R., and Mosse, C. A. (1989), A laser scanning system for the measurement of facial morphology, Optics and Lasers in Engineering, 10, 179-190.

Nechala, P., Mahoney, J., and Farkas, L. G. (1999), Digital two-dimensional photogrammetry: A comparison of three techniques of obtaining digital photographs, Plastic and Reconstructive Surgery, 103,
1819-1825.

Norusis, M. (2004), SPSS 13.0 Statistical Procedures Companion, Upper Saddle-River: Prentice Hall.

Portney, L. G. and Watkins, M. P. (1993), Foundations of clinical research: applications to practice, Stamford: Appleton and Lange.

Wickens, C. D., Lee, J. D., Liu, Y., and Gordon-Becker, S. E. (2004), An introduction to human factors engineering, 2nd Edition, Upper Saddle-River: Pearson Education. 